\title{
Minimally Invasive Detection Technologies for the Defects in Ancient Timber Structural Building
}

\author{
Peixuan Wang ${ }^{1, a}$, Wei Zhang ${ }^{1, b}$, Shengcai $\mathrm{Li}^{1, \mathrm{c}^{*}}$, Changyong $\mathrm{Hu}^{1, \mathrm{~d}}$, \\ Nicola Macchioni ${ }^{2, e}$, Jianli Yuan ${ }^{1, f}$, Youjun Zhao ${ }^{1, g}$
}

${ }^{1}$ School of Architectural Science and Engineering, Yangzhou University, Yangzhou, China, 225127

${ }^{2}$ CNR-IVALSA, Via Madonna del Piano, 650019 Sesto Fiorentino, Italy

a2385332330@qq.com, bdavidchase@163.com, ${ }^{\text {clisc@yzu.edu.cn, }{ }^{\mathrm{d}} 273230867 @ q q . c o m,}$

emacchioni@ivalsa.cnr.it, 'yjl@yzu.edu.cn, ${ }^{9981027802 @ q q . c o m ~}$

Keywords: Defects, Timber, Minimally invasion, Micro drill resistance, Stress wave

\begin{abstract}
How to examine and describe the defects in timber is vital for assessing the performance of an ancient timber structural building. This paper mainly elaborates the methodology of detecting the defects in ancient timber structures. The detection method includes preliminary condition survey, stress-wave tomography test, and correction with resistographic drilling. By means of above detection, the defects inside a timber structural building could be described more accurately.
\end{abstract}

\section{Introduction}

In a typical ancient timber structure in China, beams and columns are the main load-bearing elements. They are vulnerable to rain, worms, and their mechanical properties are easily weakened. To examine and describe the defects in timber is differcult work. Some traditional detection methods could be used to assess the surface condition of a timber structure. But they are hardly to be used to detect internal defects of timber.

At present, non-destructive test technology has developed in China and other countries. Fuwen, et al. explained the basic principle of resistographic drilling methods, gave the resistance curve form of different types of timber structural defects, and determined the evaluation methods of them [1]. Chunhui, et al. studied that how to use stress-wave tomography and resistographic drilling to test the internal defects of cylindrical timber elements [2,3]. Jian, et al. took the actual project of the Chengtang Temple in Changzi County, Shanxi Province as an example. They studied the beams and columns of ancient timber structures through the traditional detection methods, stress-wave tomography test and resistographic drilling test. At the same time, they compared the results from the two tools, then provided new ideas for the repair of ancient timber structures [4,5]. Nicola, et al. obtained the defects status and the influencing factors of the elements through the diagnosis of a church's timber structure [6].

However, little research has been conducted on the overall condition examination of a multiple floor timber structural building (such as Feiyun Pagoda shown in Fig. 1). In this paper, on the basis of predecessors' experiences, stress-wave tomography and resistographic drilling are combined togather to detect the internal defects of timber structures so that a detailed investigation and analysis of related defects could be clearly presented.

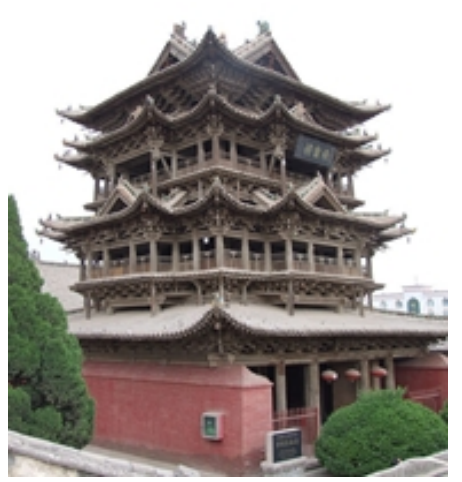

Fig. 1 Feiyun Pagoda

\section{Methodologies and Techniques}

With the conservation of heritage buildings which contain timber elements, the main aim is to guarantee that condition survey and assessment provide the necessary data for historical analysis, structural safety assessment and planning of intervention works. The procedure required for the on-site examination and assessment of an historic timber structure is as follows(Fig. 2). 


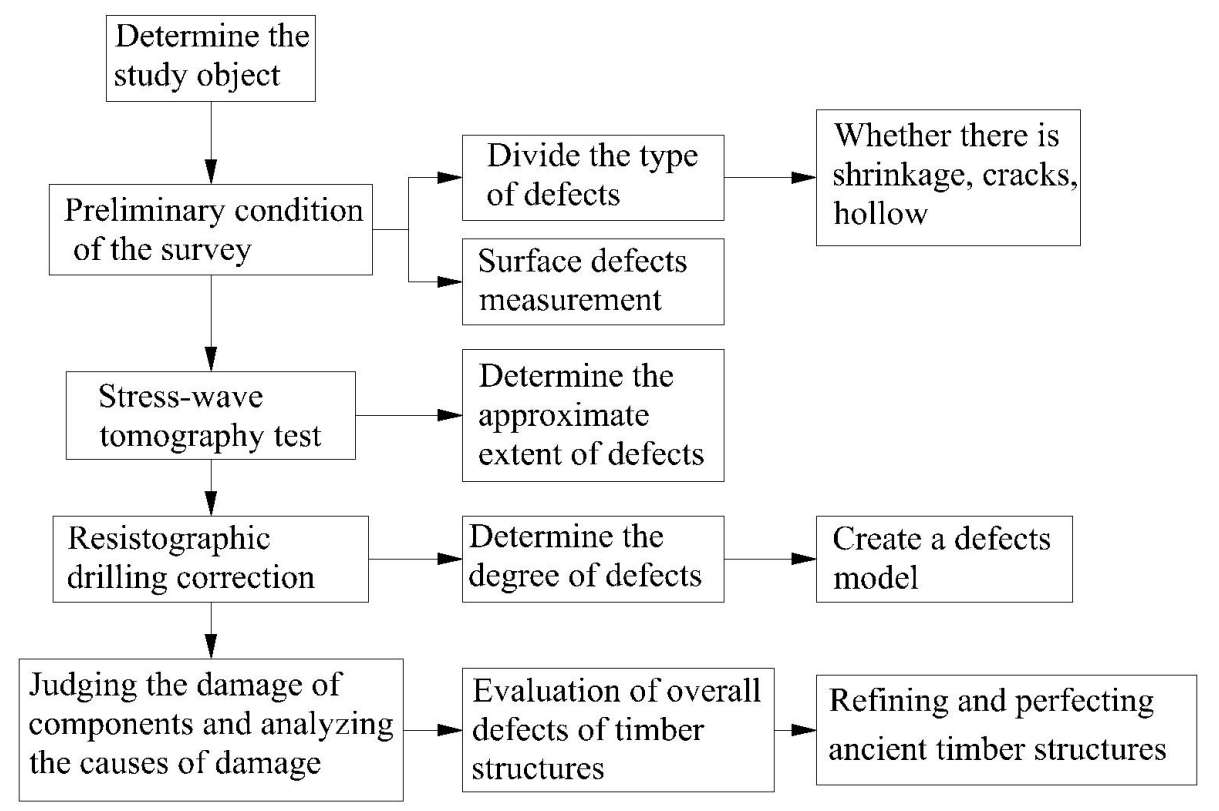

Fig. 2 Road map of timber structures inspection technology

Preliminary condition of survey. Due to its own particularities, some serious defects can be directly observed by the naked eye. They are decay, cracks, and shrinkage. In the detection of ancient timber structures, the defects of columns, beams, and roofs should be mainly recorded as cracks. It will be classified into four categories according to the length and width of the cracks. Type I is a component without obvious cracks, and its elements are basically in good condition; Type II includes the cracks whose width is no more than $10 \mathrm{~mm}$ and the length is no more than $500 \mathrm{~mm}$. Type III is the cracks whose width is between $10-25 \mathrm{~mm}$ and the length is between $500-1500 \mathrm{~mm}$. Type IV is the cracks whose width over $25 \mathrm{~mm}$ and the length is over $1500 \mathrm{~mm}$.

Secondly, the depth of defects which is on the columns, beams, and Dou-gong sets of each floor could be measured. When one column is diagnosed, one point in each direction of the east, south, west, and north should be chosen, from the height of $0.50-0.15 \mathrm{~m}$ above the ground, then to drill in and measure the penetration depth with a probe. Similarly, when studying beams and Dou-Gong sets, the points inside and outside evenly are picked up and probe depth of points in different directions. What is important is that deep corrosion area should be kept away when selecting the measuring points. Universal and representative parts might be better than others.

Stress-wave tomography test. The stress-wave tomography exploits the internal bias of the timber particles and the principle that the cavity caused by the degradation of the material leads to a slower propagation time. The 'defects' area can be observed without damaging the elements. The sensor is fixed at a distance around the circumference of the timber elements and then each sensor were hammered in. The stress waves are generated by the tapping propagate along the internal of the timber and received by other sensors. The diffusion time is recorded. Different stress wave propagation paths lead to different propagation times. Based on the test data, the instrument software generates a two-dimensional reconstruction map or a line segment map. Three sections of different heights on each column were measured. 12 sensors were installed in the same section and all of them are at the same angle with each other (Fig. 3). Similarly, different cross-sections on the beams were selected to be detected and corresponding inspection images were generated.

The image generated by the software will indicate the defects degree through different colors. However, according to similar tests conducted by the laboratory, it is found that sometimes the "defects" showed by software is actually a shrinkage crack. These cracks show the discontinuity of the grain in the transverse direction, but they cannot be called "defects" because they are inevitable. At the same time, they do not reduce the mechanical properties of the timber elements, especially in the column structures. Therefore, relying solely on the detection of the stress-wave tomography is 
impossible to accurately locate and classify the internal defects of the timber elements. The results must be corrected in conjunction with other non-destructive test methods.

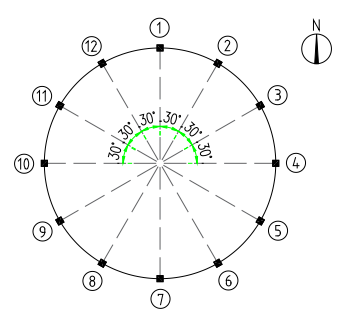

(a) The angle of sensors

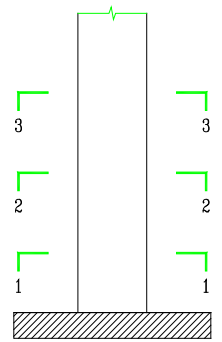

(b) Detected sections of column

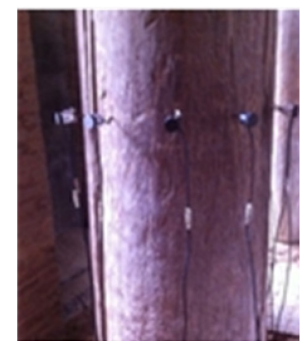

(c) The installation of sensors

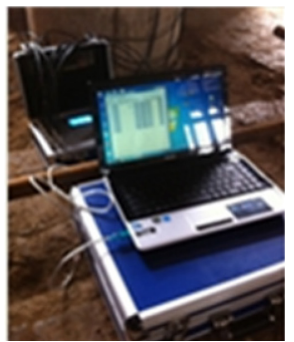

(d) Software generated image

Fig. 3 Stress-wave tomography test

Resistographic drilling correction. The working principle of the resistographic drilling is to drive the drill needle into the interior of the timber at a constant rate through the motor drive. During the drilling process, corresponding resistance will be generated. According to the resistance curve map and relevant knowledge of the timber, the internal decay of the timber can be judged. During the inspection process, the length of the resistance gauge needle is set slightly larger than the timber diameter to reduce unnecessary wear and time. For the

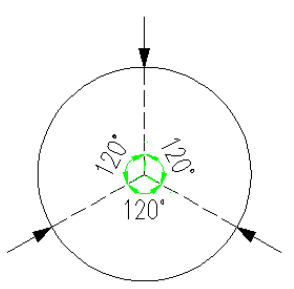

Fig. 4 Inspection points in the same section same column, the three sections examined by the stress-wave tomography are the detection sections. For the same inspection section, three different directions were chosen to inspect, and the three directions are at $120^{\circ}$ with each other (Fig. 4).

After the end of the test, the specific internal defects of the timber elements can be determined according to the spectrum displayed by instrument. Under normal circumstances, the resistance curve of the timber elements without defects is more stable and uniform. Its frequency is faster, the cycle is stable, and the peak-wave trough phenomenon continuously appears. When there are decayed or worm-eaten areas in the timber structures, the resistance curve will fluctuate. Its decrease will be more obvious, and there is a gentle slope area in the process. When there are cracks in the timber elements, the curve will drop steeply, and shows a cliff. When resistographic drilling is performed, the resistance curve will suddenly rise from the normal level when there is a knot in the timber elements, and the overall level is equivalent to several times of the normal level. In addition, the timber knots will affect the resistance curve after them, which will result the upward shift of the resistance curve. Even the curve of the decayed part may be showed as a normal level.

\section{Case Study}

Feiyun Pagoda has three service floors and two structure floors. Its plane is square, the middle plane becomes a cross, and the corridor is outside. It is beam-column system, and the beam-column is mostly connected by Dou-gong sets.

Preliminary survey. In order to facilitate the detection and recording, the columns of each floor are numbered from the southwest corner. the fracture parameters were recorded, the type of defects were divided, and the defects distribution of each floor were summarized (Fig. 5, Fig. 6). Different floors have different degrees of defects. Overall, the defects of the columns in the first floor is most serious. Compairing the degree of defects in the columns of each floor, the permanent columns whose diameter is $270 \mathrm{~mm}$ have been decayed the most serious. From the maximum direction of cracks in each column, the defects is most serious on the east and west sides of the columns, followed by the south and the north. 


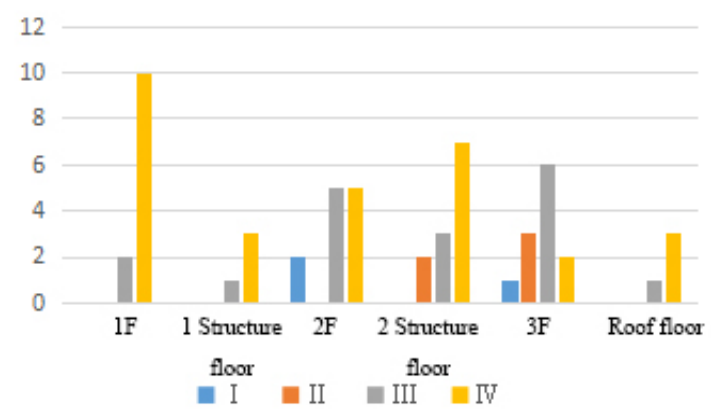

Fig. 5 Defects summary of columns in each floor

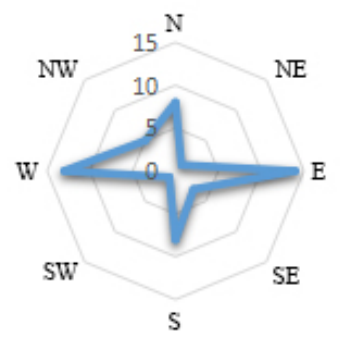

Fig. 6 Defects distribution of each column

The defects of beams is mainly based on cracks. The development of these cracks severely impairs the bearing capacity of beams. When the external load exceeds the bearing capacity of the elements, the elements will be defected. The beams of each floor is numbered from the lowest one in the south, and the fracture parameters of each beam are recorded. The defects were classified, and the defects directions of each beam were summarized( Fig. 7, Fig. 8). The beams on each floor of the Feiyun Pagoda also suffered from varying degrees of defects. The first floor and the third floor were most decayed. From the perspective of the maximum cracks in each beam, the degree of defects in the four directions of the east, west, south, and north is basically the same.

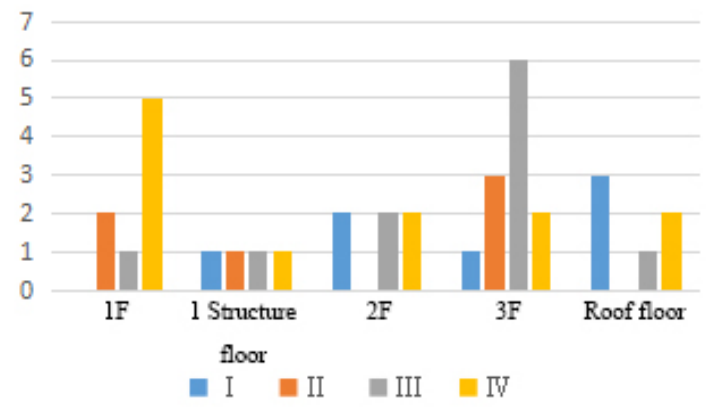

Fig. 7 Defects summary of beams in each floor

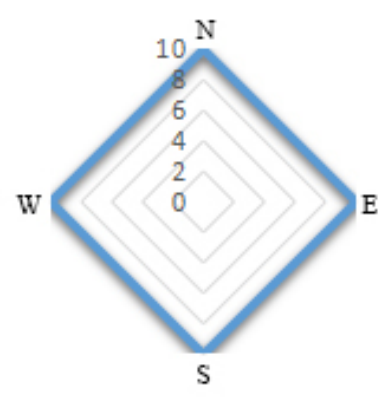

Fig. 8 Defects distribution of each beam

The surface defects data is summarized and plotted as a line graph (Fig. 9, the first floor was taken as an example). The average defects depth in the columns of the first floor is about $2.8 \mathrm{~mm}$, and the maximum is about $9.0 \mathrm{~mm}$. The average depth of defects in the columns of the second floor is $2.5 \mathrm{~mm}$, and the maximum is approximately $12.0 \mathrm{~mm}$. On the third floor, the average defects depth is about $1.4 \mathrm{~mm}$, and the maximum is about $6.0 \mathrm{~mm}$. For beams on the second floor, the average defects depth is $1.0 \mathrm{~mm}$; the maximum is close to $4.0 \mathrm{~mm}$. For Dou-gong sets, the average depth of defects on the east side is about $1.3 \mathrm{~mm}$, the depth in south side is about $0.9 \mathrm{~mm}$, the depth in west side is about $1.7 \mathrm{~mm}$, and the depth in north side is about $1.8 \mathrm{~mm}$. In general, the southern Dou-gong sets suffered less defects, while the northern side of the Dou-gong sets was most heavily defectsd, even close to $6.5 \mathrm{~mm}$.

Stress-wave tomography test. Based on the data obtained by stress-wave tomography, the percentage of defects area per section was calculated and the average of the three section percentages of the same column was taken to compare (Fig. 10). Except the highest percentage of decay area in the columns 1 and 11 , the overall quality of the remaining columns is good, at least within the investigated height $(0.50-0.15 \mathrm{~m})$. For the two columns with the highest percentage of "defects" areas (column 1 and column 11), the column 11 was choosen as an example to conducte a detailed analysis of the 2D reconstruction chart of each section (Fig. 11). 


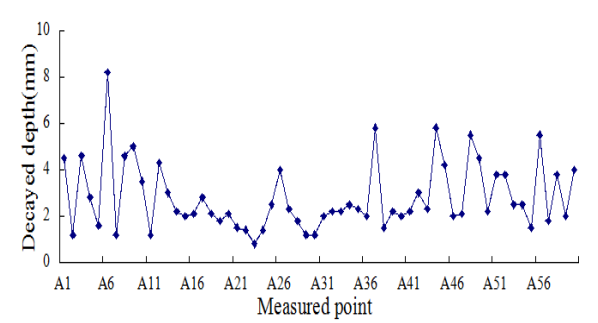

Fig. 9 Defects depth of the first floor

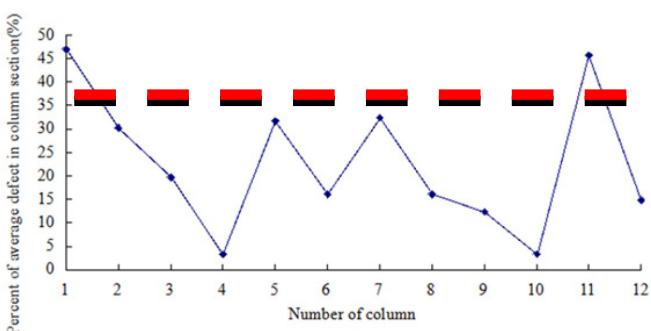

Fig. 10 Percentage distribution of defects in columns of the first floor

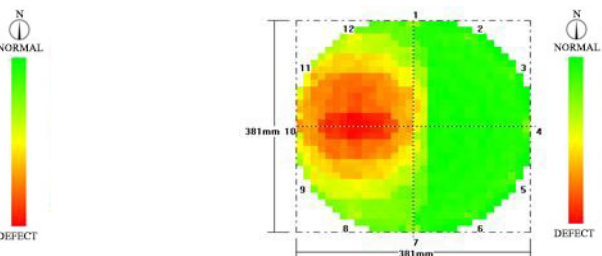

(c)

(a)

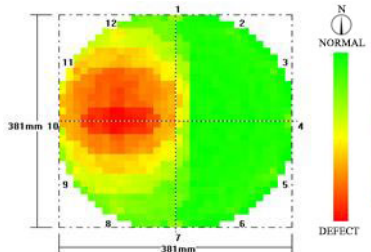

(b)

Fig. 11 2-D reconstruction chart of column $11(\mathrm{a}, \mathrm{b}, \mathrm{c})$

(Colors from green to red indicate defects from slight to severe)

Resistographic drilling correction. The test examined all the columns of the first, second and third floor. However, due to the limitation of on-site conditions, the beams and Dou-gong sets were tested only on the second floor.

Because of the limitations of the article space, all the test data cannot be expressed. Here, the a section of the column 11 in the first floor with more severe defects is taken as an example for detailed analysis. The resistographic drilling test was performed on the column 11 from three measuring points, and the following spectrum was obtained. From the spectrum followed, it was observed that the resistance almost dropped to 0 in the range of $19-23 \mathrm{~cm}, 22-40 \mathrm{~cm}$, and $6-15 \mathrm{~cm}$, and it can be judged that there was defects here (Fig. 12, Fig. 13, Fig. 14). By connecting these measuring points in a certain way to form areas, the defects range can be displayed in more detail (Fig. 15). At the same time, it can be observed that there is a momentary decrease in the resistance value at about $28 \mathrm{~cm}$ in fig. 14 , and the range of the fall is very small. Combining with the stress-wave tomography, it is not defects. It may be caused by timber shrinkage which is the normal phenomenon of cracks. Using the same judgment method, the defects range of the column 11 on the $b$ and $c$ sections was obtained. Then a three-dimensional defects model (Fig. 16) was created by the spectrum.

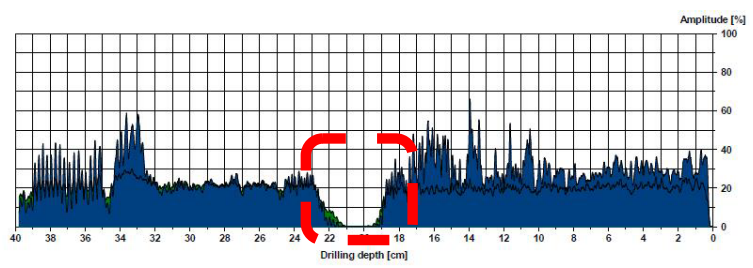

Fig. 12 Point 1 of column 11-a

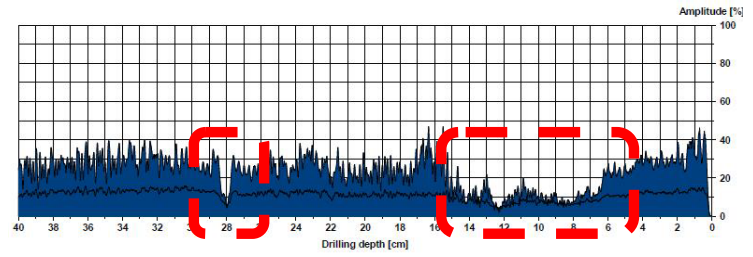

Fig. 14 Point 3 of column 11-a

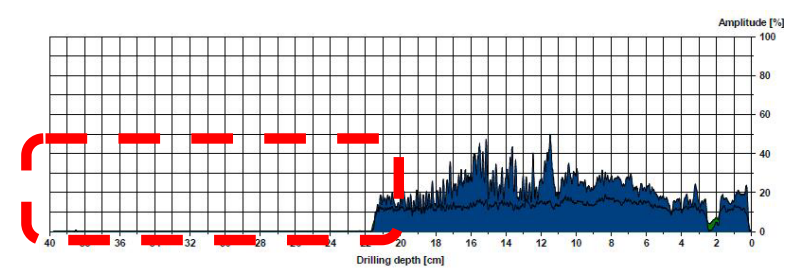

Fig. 13 Point 2 of column 11-a

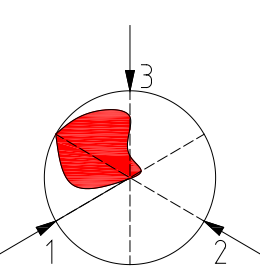

Fig. 15 Defects of column 11-a

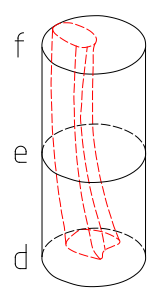

Fig. 16 3D defects model of column 11

Analysis. Combining all the studies that have been conducted, the different defects levels of beams, columns, and Dou-gong sets according Table 1 (Fig. 17) can be classified. The results of the study showed that the defects of timber structures in the Feiyun Pagoda was concentrated on the first and 
the second levels, and most of them were on the first level. Therefore, it is concluded that most of the timber structures of Feiyun Pagoda have slight defects, but the overall performance is basically intact.

Table 1 GB/T13942--92 Classification Criteria for

Timber Corrosion Resistance

\begin{tabular}{cl}
\hline $\begin{array}{c}\text { Defects } \\
\text { level }\end{array}$ & \multicolumn{1}{c}{ Defects degree } \\
\hline 0 & $\begin{array}{l}\text { The material is intact and there is no } \\
\text { defects state under the naked eye }\end{array}$ \\
1 & $\begin{array}{l}\text { The surface has a slight defects } \\
2\end{array}$ \\
& $\begin{array}{l}\text { Medium defects, depth is between } \\
\text { 2-5mm, area is up to } 1 / 3\end{array}$ \\
3 & $\begin{array}{l}\text { Serious defects, depth is between } \\
5-10 \mathrm{~mm}, \text { area is up to } 2 / 3\end{array}$
\end{tabular}

4 it can be easily broken

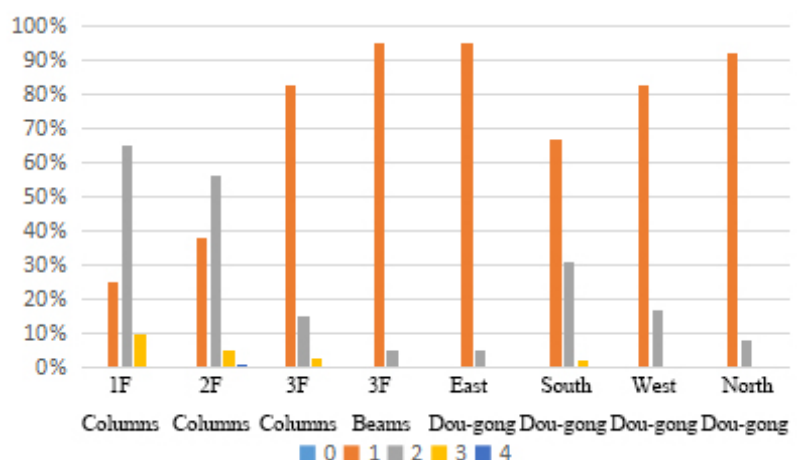

Fig. 17 Distribution of Defects Levels of structures of Feiyun Pagoda

\section{Results and Discussion}

Research shows that load-bearing elements of Feiyun Pagoda basically have a certain degree of minor defects, a few of them are moderately rot, and fewer of them are very corrupt. The overall defects degree of the timber elements on the north side of Feiyun Pagoda is the largest, while the defects on the south side is the smallest. The reason for such an assumption is that the north side of the pagoda has been kept wet for a long period of time, and it has received less sunlight, which has grown pests and bacteria.

Due to the wall covering on two sides of the Feiyun Pagoda, the ventilation is poor, so the defects degree of the timber elements in the first floor is the greatest. Because of the rising pagoda height, the timber elements in the second and the third floor have better ventilation and sunshine, and the degree of timber structural defects has been gradually reduced. On the same floor, the defects of the columns is mainly concentrated on the base of them, because the base of the column is vulnerable to flooding of the floor. Compared with columns, beams and Dou-gong sets have less defects, because they are less likely to come into contact with the moisture of the floors' surface.

In the repair and protection work of the Feiyun Pagoda, the elements which were slightly decayed will be reinforced, and the moderate defects elements should be done appropriate demolition and replacement. During the repair process, special attention should be paid to elements that are prone to moisture and poor ventilation. These elements are generally located on the base of columns, outside of pagoda and roofs.

\section{Conclusion}

The article analyzes the defects degree of Feiyun Pagoda in Wanrong County, Shanxi Province, proposes measures for repairing and protecting timber structures based on the analysis results. The three scholars Guanfeng Qiao, Tieying Li, and Xiaoli Wang wrote three articles, they are "Study on Current Situation Assessment and Reinforcement Design Scheme of Feiyun Pagoda", " Repair and Reinforcement of Feiyun Pagoda", and "Study on Rapid Inspection and Identification of Decayed Elements of Ancient Timber Structures" [7,8,9]. These articles have already described the exploration of Feiyun Pagoda in detail. In these articles, preliminary condition of the survey, stress-wave tomography test, resistographic drilling correction and other methods were also used. However, in the course of the study, the connection between them was not established. In this paper, the results of the these technologies are combined to make the defects of timber structures judgment concrete. At the same time, the defects location is more intuitively marked by means of three-dimensional model. 
This article summarizes a set of methods for evaluating the defects of ancient timber structures: preliminary condition of the survey, stress-wave tomography test, Resistographic drilling correction , 3D model analysis. This detection technology can further clarify the defects position of the timber structures and determine the exact degree of defects.

Of course, there are still deficiencies in the research process. Some structures such as columns, beams, and Dou-gong sets were selected for test in the experiment, but the results obtained may not represent the degree of defects of the entire elements. Due to the limitations of the structure and geographical conditions, some timber elements of the case were not be tested. Some of the research results were derived through data and experience. The accuracy of the study remains to be further verified.

\section{Acknowledgements}

This paper is supported by the National Natural Science Foundation of China (Grant No. 51478409, 51338001), the State Administration of Foreign Experts Affair (Grant No. GDT20163200026), Program of 100 Foreign Experts in Jiangsu Province (Grant No. JSB2017029)

\section{References}

[1] Fuwen Zhang, Qingfeng Xu, Zhiyu Zhang, Qiong Liu, Resistographic Drilling Method to Detect Timber defects [J], Non-destructive Test, 2016, 38(01): 6-9+74.

[2] Chunhui Liao, Study on Non-destructive Screening Methods for Internal Defects of Timber Elements in Ancient Buildings [D], Beijing Forestry University, 2015.

[3] Chunhui Liao, Houjiang Zhang, Dongqing Li, Yanliang Sun, Xiping Wang, Study on Screening Method of Internal Defects in Cylindrical Timber Elements of Ancient Buildings [J], Journal of Beijing Forestry University, 2013, 35(01): 123-126.

[4] Jian Dai, Lihong Chang, Wei Qian, Hao Chang, Non-destructive Test Method and Application of Internal Defects in Ancient Timber Elements [J], Journal of Architecture, 2017(02): 7-10.

[5] Jian Dai, Lihong Chang, Wei Qian, Xin Li, Study on Non-destructive Test and Appliation of Defects Characteristics of Timber Elements in Ancient Buildings [J], Journal of Beijing University of Technology, 2016, 42(02): 236-244.

[6] Nicola Macchioni, Michele Brunetti, Benedetto Pizzo.The Timber Structures in the Church of the Nativity in Bethlehem: Typologies and Diagnosis [J]. Journal of Cultural Heritage, 2012, 8: 42-53.

[7] Guanfeng Qiao, Tieying Li, Study on Current Situation Assessment and Reinforcement Design Scheme of Feiyun Pagoda [J], Industrial Building, 2014, 44(10): 170-175.

[8] Guanfeng Qiao, Tieying Li, Repair and Reinforcement of Feiyun Pagoda [J], Industrial Building, 2016, 46(09): 175-178+121.

[9] Xiaoli Wang, Study on Rapid Inspection and Identification of Defectsd Elements of Ancient Built Timber Structures [D], Beijing Jiaotong University, 2017. 